OPEN ACCESS

Edited by:

Misha Vorobyev,

University of Auckland, New Zealand

Reviewed by:

Linda Isaac,

University of California,

San Francisco, United States

Quoc Vuong,

Newcastle University, United Kingdom

*Correspondence:

Qi Zhang

zhangqikashi@163.com

Specialty section:

This article was submitted to

Perception Science,

a section of the journal

Frontiers in Psychology

Received: 25 February 2018

Accepted: 28 August 2018

Published: 28 September 2018

Citation:

Zhang Q, Ran G and Li X (2018) The Perception of Facial Emotional

Change in Social Anxiety: An ERP

Study. Front. Psychol. 9:1737.

doi: 10.3389/fpsyg.2018.01737

\section{The Perception of Facial Emotional Change in Social Anxiety: An ERP Study}

\author{
Qi Zhang ${ }^{1 *}$, Guangming Ran' and Xueping $\mathrm{Li}^{1}$ \\ ${ }^{1}$ College of Preschool and Primary Education, China West Normal University, Nanchong, China, ${ }^{2}$ Department \\ of Psychology, Institute of Education, China West Normal University, Nanchong, China
}

Social anxiety is one of the psychological symptoms that most commonly occur in social interaction. Although previous behavioral studies have investigated the neutral-angry facial emotion change in social anxiety, none of the previous studies have, however, directly investigated the angry-neutral facial emotional change. Furthermore, less is known about the neural correlates of the facial emotion changes in individuals with social anxiety. The main goal of the present study was to explore the perception of facial emotional changes in individuals with social anxiety, using high temporal resolution event-related potential techniques. Behaviorally, accuracy in the angry-neutral facial emotional change trail was lower than that in the neutral-neutral case. Neurally, we found that the N170 amplitudes in angry-neutral facial emotional change trial were larger than those in the neutral-neutral case for high social anxiety (HAS) participants, probably reflecting that they might engage in more analytical processing of different facial elements. Interestingly, HSA participants showed smaller P200 left hemisphere amplitudes in the angry-neutral facial emotional change trial when compared with the neutral-neutral case, which suggested that they might have difficulties in processing emotions when they encounter these facial emotional changes. Finally, the late positive potential amplitudes in the neutral-angry and angry-neutral facial emotional change trials were smaller than those in the neutral-neutral case, regardless of the social anxiety. These results suggest that social anxiety influences the facial emotional changes mainly at an earlier stage of processing.

Keywords: angry-neutral facial emotional changes, neutral-angry facial emotional changes, social anxiety, perception, ERP

\section{INTRODUCTION}

Social anxiety refers to a state of anxiety that results from the presence of interpersonal evaluation in social interaction (Pierce, 2009). Although social anxiety is a milder form of social phobia, it is one of the most common forms of anxiety (Pierce, 2009; Kross et al., 2014). It is associated with poor interpersonal relations, such as negative, aversive, or exclusionary experiences with others (Mathew et al., 2011). It begins during one's childhood or adolescence, with a mean age of onset between 14 and 16 years (Iverach and Rapee, 2014). Individuals with high social anxiety (HSA) show enhanced vigilance to stimuli associated with social threat, such as angry faces (Kirsch, 2015). 
As fundamental emotional stimuli, emotional faces play a significant role in our daily life (Ran et al., 2014b). A large number of electrophysiological studies have explored the perception of emotional faces in individuals with social anxiety. An early event-related potential (ERP) component is P100, which is probably generated by the extrastriate cortex (Campanella et al., 2006). Such a component is a positive wave that is recorded around $100 \mathrm{~ms}$ following the stimulus presentation onset at occipital sites (Fishman et al., 2012). The P100 component is thought to reflect early visual processing (Schendan et al., 1998; Taylor and Khan, 2000). A set of ERP studies on facial processing in individuals with HSA showed increased P100 amplitudes in response to threatening/angry faces (Mühlberger et al., 2009; Peschard et al., 2013). Interestingly, Rossignol et al. (2012) reported enhancement of the P100 amplitudes in individuals with HSA for all emotional faces rather than a specific increase in response to threatening faces.

Unlike the P100 component, the N170 component (a negative wave), is recorded at occipitotemporal sites peaking roughly at $170 \mathrm{~ms}$ after stimulus onset (Bentin et al., 1996; Chen et al., 2015). A meta-analysis of N170 shows that this negative component is usually observed in experiments that use faces as stimuli (Hinojosa et al., 2015). It has previously been argued that N170 reflects the analytical processing of different facial elements (Peschard et al., 2013). There is evidence that N170 is larger (more negative amplitudes) for HSA individuals when they perform a task that measures the processing of emotional faces that are threatening (Wieser et al., 2010; Ran and Chen, 2017). However, several other studies have failed to find the moderating effect of social anxiety on N170 (Mühlberger et al., 2009; Li et al., 2017). These discrepancies might stem from differences in participants' task and experimental design. In the study conducted by Mühlberger et al. (2009), the processing of emotion was implicit. However, participants were explicitly instructed to process emotion in the study conducted by Ran and Chen (2017). More precisely, HSA and low social anxiety (LSA) participants attend to emotion in the explicit task, while they do not attend to emotion in the implicit task, which may affect their configural encoding of emotional faces.

The P200 component, which follows N170 and is recorded at occipitotemporal sites, is the second positive peak in ERP, occurring at around $190 \mathrm{~ms}$ to $250 \mathrm{~ms}$ after stimulus onset (Sheehan et al., 2005). It is believed to reflect sustained perceptual processing and to be functionally associated with the complexity of emotional appraisal (Peschard et al., 2013). Previous studies that examined the effect of social anxiety on P200 demonstrated increased P200 amplitudes in response to angry faces in HSA individuals (Rossignol et al., 2012). Recently, Ran and Chen (2017) reported that HSA participants exhibited more positive P200 amplitudes in response to angry faces when compared with happy faces.

A late ERP component, which is sensitive to emotional faces, is the late positive potential (LPP) that starts around $300 \mathrm{~ms}$ after stimulus onset (Sje and van Strien, 2018). The LPP is a centro-parietal distributed and a positive-going ERP component (Sje and van Strien, 2018). There is evidence that the LPP is larger for emotional stimuli when compared with neutral stimuli (Hagemann et al., 2016). A particular study observed enhanced LPP amplitudes in HSA participants when they viewed angry and disgusted faces (Moser et al., 2008). In addition, a study that explored the affective context found that faces in negative contexts elicit larger LPP amplitudes when compared with faces in neutral contexts in HSA participants (Wieser and Moscovitch, 2015).

Human beings always encounter dynamic but not static emotional faces in daily life (Zhang et al., 2015). Changes in emotional faces are more important to social communication than static emotional faces (Zhang et al., 2015). An earlier study used a morphed faces task to assess participants' identification biases (Joormann and Gotlib, 2006). The following study adopted the modified morphed faces task to examine dynamic facial expressions in individuals with social anxiety (Heuer et al., 2010). Recently, Gutiérrez-García and Calvo (2017) found that HSA participants were biased towards the interpretation of ambiguous expressions (dynamic facial expressions) in response to threat. Although previous behavioral research has investigated the facial emotional change (neutral-angry facial emotional change: a neutral face suddenly changes to an angry face) in individuals with social anxiety, none of the previous studies have directly investigated the angry-neutral facial emotional change (an angry face suddenly changes to a neutral face). Furthermore, less is known about the neural correlates of the facial emotional changes in individuals with social anxiety.

The main goal of the present study is to explore the perception of facial emotional changes in individuals with social anxiety, using high temporal resolution ERP techniques. As HSA participants show more negative N170 amplitudes when they process threat stimuli (Ran and Chen, 2017), we predict increased N170 amplitudes in the angry-neutral facial emotional change trials. Considering the fact that HSA participants might show an inferior recognition performance with emotional appraisal when they encounter the angry-neutral facial emotional change (Zhang et al., 2015), we hypothesized that they would exhibit decreased P200 amplitudes in the angryneutral facial emotional change trials. Finally, we expected the LPP amplitudes in the neutral-angry and angry-neutral facial emotional change trials to be smaller than those in the neutralneutral case.

To test these hypotheses, we adopted a S1-S2 paradigm, which was used by previous researchers to explore the recognition of emotional faces (Ran et al., 2014a, c; Li et al., 2017). Participants with HSA and LSA were instructed to perform a task of facial identity recognition, and their brain responses were recorded using high temporal resolution ERP techniques. It was noted that the participants in present study were students elevated on a scale. They were not clinically diagnosed with social anxiety disorder by a clinician such as a psychiatrist. In this paper, we focus on P100, N170, P200, and LPP components, as it has been proposed that these components are relevant for emotional faces and social anxiety (Peschard et al., 2013; Hagemann et al., 2016; Ran and Chen, 2017). 


\section{MATERIALS AND METHODS}

\section{Participants}

Twenty-seven healthy volunteers (15 women and 12 men; mean age $=21.30$ years, standard deviation $=1.84$ years) participated in the experiment. They were right-handed and had normal or corrected-to-normal vision. All participants were preselected from a group of 925 undergraduate students based on their social anxiety scores on the Chinese version of the Liebowitz Social Anxiety Scale (LSASC) (He and Zhang, 2004). According to a recent ERP study on individuals with social anxiety (Ran and Chen, 2017), the HSA participants ( $N=13,7$ women) were defined as those who scored 60 or greater on the LSASC, while the LSA participants ( $N=14,8$ women) were those who scored under 40. To control comorbidity, all participants were asked to complete the Spielberger State-Trait Anxiety Inventory (Spielberger et al., 1983) and the Beck Depression Inventory (Beck and Beamesderfer, 1974).

It was found that the HSA and LSA participants differed in the LSAS-SR total scores (HSA: $77.23 \pm 15.77$, LSA: $26.07 \pm 6.97$; $t(25)=-11.05, p<0.001)$, but no group differences were found for age (HSA: $21.16 \pm 1.62$, LSA: $21.43 \pm 2.07 ; t(25)=0.38$, $p=0.706$ ), state anxiety level (HSA: $42.08 \pm 6.46$, LSA: $38.14 \pm 7.79 ; t(25)=-1.42, p=0.168)$, trait anxiety level (HSA: $44.23 \pm 7.81$, LSA: $40.36 \pm 9.39 ; t(25)=-0.86, p=0.397)$, and depression (HSA: $11.77 \pm 6.11$, LSA: $8.00 \pm 5.88 ; t(25)=-1.63$, $p=0.115)$. All participants provided written informed consent and received course credit for their participation. The study was approved by the local ethics committee and the experiments were carried out in accordance with the approved guidelines.

\section{Materials}

Following previous studies (Zhang et al., 2017), the present study adopted three different videos of facial emotional changes (angryneutral videos (Figure 1A): an angry face changes to a neutral face, neutral-angry videos (Figure 1B): a neutral face changes to an angry face, neutral-neutral videos (Figure 1C): a neutral face changes to a neutral face). We used a set of 78 videos (26 angryneutral videos, 26 neutral-angry videos, and 26 neutral-neutral videos). The videos were created by using the photo-morphing software (Abrosoft FantaMorph). To acquire the neutral-angry videos, pairs of static images displaying a neutral expression (the first image) and an angry expression (the last image) provided reference points for creating a sequential morph between the two images. Sequential morphs were compiled into $600 \mathrm{~ms}$ video files (AVI format) with a frame rate of 15 frames per second. The pairs of static images (the first image: an angry expression, the last image: a neutral expression) were employed to create the angryneutral videos, and the pairs of static images (the first and last image: a neutral expression) were used to acquire the neutralneutral videos. The faces in the videos had the same ethnicity (Chinese). One half of the videos displayed female faces, while the other half displayed male faces. A group of volunteers, who did not participate in our experiment, were recruited to rate the valence and arousal of the 78 videos on a 9 -item scale $(1=$ low pleasure, low arousal; 9 = high pleasure, high arousal). The videos
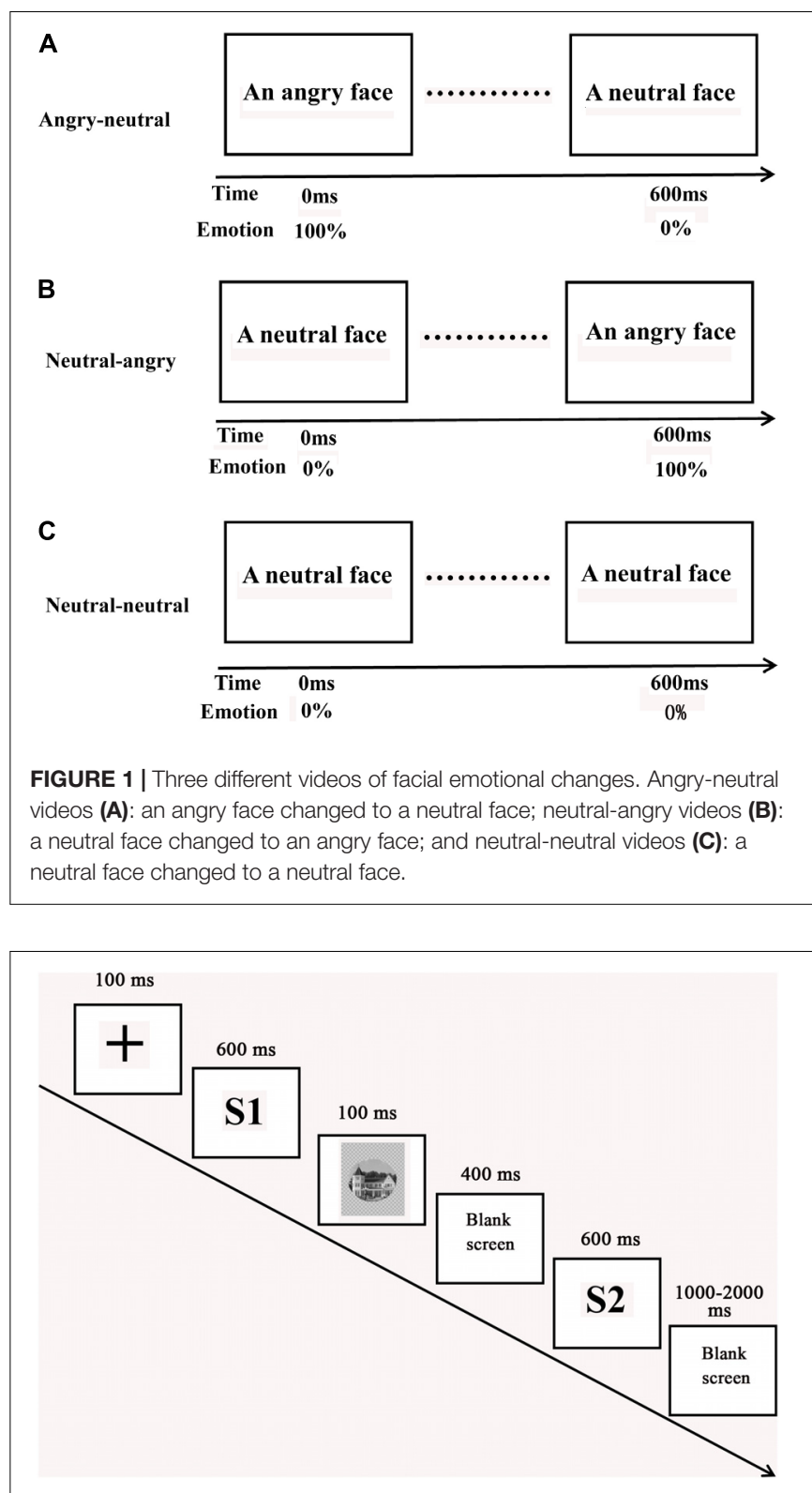

FIGURE 2 | Schematic illustration of the experimental procedure.

differed significantly in the valence dimension $(p<0.001)$ but were similar in arousal $(p=0.106)$. The materials also consisted of eight house pictures that were mask stimuli.

\section{Procedure}

Participants were seated comfortably at a position that was $90 \mathrm{~cm}$ in front of the computer screen and were instructed to try their best to avoid head movements and eye blinks. The experimental procedure was organized by E-PRIME 2.0. The current experiment employed the S1-S2 paradigm, which was used by previous researchers to explore the recognition of emotional faces (Ran et al., 2014a, c). On each trial of the experiment (Figure 2), a fixation cross was shown for $100 \mathrm{~ms}$. 
After the cross disappeared, the first facial emotional change video (S1) was presented for $600 \mathrm{~ms}$. Next, a stimulus of house picture (a mask stimulus) was depicted for $100 \mathrm{~ms}$. Following a blank screen (400 ms), the second facial emotional change video (S2) appeared for $600 \mathrm{~ms}$. Finally, a blank screen terminated the trial, which lasted for 1000-2000 ms. The participants were asked to judge the identity of the face in the videos during the 1000-2000 ms blank interval after S2. The S1 and S2 videos were the same expression change in each trial. Participants completed two blocks of 90 trials, yielding a total of 180 trials per participant (60 angry-neutral facial emotional change trials, 60 neutral-angry facial emotional change trials, and 60 neutralneutral facial emotional change trials). There were equal numbers of same and different trials. Before the experiment, participants received instructions and performed a block of six practice trials that included two practice trials for each expression change condition.

\section{EEG Recording and Analysis}

Electroencephalogram (EEG) was recorded from 64 scalp sites using the BrainAmps system (Brain Products, Munchen, Germany). The vertical electrooculogram (EOG) was recorded with electrodes placed below the right eye and the horizontal EOG was recorded from the right orbital rim. Electrode impedance was held below $5 \mathrm{k} \Omega$. The EEG and EOG activities were amplified using a DC-100 Hz bandpass and were continuously sampled at $500 \mathrm{~Hz} / \mathrm{channel}$.

For preprocessing the ERP analysis, the EEG data were recomputed to average mastoid reference. The data were further filtered off-line (0.05-30 Hz bandwidth). Ocular artifacts (blinks and other movements) were corrected using a Gratton and Coles-based algorithm off-line. Trials with artifacts exceeding $100 \mu \mathrm{V}$ mainly due to amplifier clippings and peak-to-peak deflections were omitted from the averaging. The EEG data were segmented from $-200 \mathrm{~ms}$ to $1000 \mathrm{~ms}$ relative to the second facial emotional change video (S2), with a $200 \mathrm{~ms}$ prestimulus baseline. Based on previous studies (Luo et al., 2010; Caharel et al., 2011; Ran et al., 2014c), the P100 component (80-130 ms) was analyzed at O1/O2, P3/P4, and PO3/PO4 electrodes. In addition, the N170 component was analyzed within a time frame of $130-190 \mathrm{~ms}$ post stimulus onset at P5/P6, P7/P8, and PO7/PO8 electrodes. The P200 component (190-250 ms) was determined over O1/O2, P3/P4, PO3/PO4, and PO7/PO8 electrodes. Peak amplitudes and latencies of these components were subjected to repeated-measures ANOVA with facial emotional changes (angry-neutral, neutral-angry, vs. neutral-neutral) and hemisphere (right vs. left) as withinparticipant factors and with social anxiety (high vs. low) as a between-participant factor.

Given the absence of a sharply defined peak (Zhang et al., 2006; Van Beek et al., 2014), the LPP (300-700 ms) component was observed and quantified as mean amplitudes at the following sites: $\mathrm{C} 3 / \mathrm{C} 4, \mathrm{P} 3 / \mathrm{P} 4$, and $\mathrm{Cz} / \mathrm{Pz}$ electrodes (Hagemann et al., 2016). Mean amplitudes of the LPP component were entered into a $3 \times 3 \times 2$ ANOVA with the within-participant factors facial emotional changes, hemisphere (left, middle, vs. right), and the between-participant factor social anxiety. When appropriate, degrees of freedom for the repeated-measures factors were corrected according to Greenhouse-Geisser.

\section{RESULTS}

\section{Behavioral Results}

A repeated-measure ANOVA with facial emotional changes (angry-neutral, neutral-angry, vs. neutral-neutral) as a withinparticipant factor, and social anxiety (high vs. low) as a betweenparticipant factor was calculated based on participants' accuracy and reaction time (RT) data. The mean accuracy and RT for each condition are displayed in Table $\mathbf{1 .}$

The analysis of the accuracy data showed a main effect for facial emotional changes $[F(2,50)=23.50, p<0.001$, $\left.\eta_{\mathrm{p}}^{2}=0.484\right]$. Post hoc $t$-tests showed that the accuracy rates in the angry-neutral facial emotional change condition were lower $(M=86.04 \%, S D=7.77 \%)$ than those in the neutral-neutral $(M=93.17 \%, S D=5.43 \%)$ facial emotional change condition $[t(26)=-5.31, p<0.001]$. However, the differences in accuracy rates between the angry-neutral $(M=86.04 \%, S D=7.77 \%)$ and neutral-angry $(M=83.57 \%, S D=10.47 \%)$ facial emotional change trials failed to reach significance $[t(26)=1.68, p=0.104]$. With regard to the RT data, no significant effects were found (all Fs $<3.17$, ps $>0.051$ ).

To see if S1 had an influence on S2, we compared the accuracy and RT data in same vs. different trials. We found that the accuracy rates in the same trial $(M=96.91 \%, S D=2.99 \%)$ were higher than those seen in the different $(M=76.12 \%$,

TABLE 1 | Means and standard deviations of accuracy and reaction time (RT) for HSA (high socially anxious) and LSA (low socially anxious) group in each facial emotional change condition.

\begin{tabular}{|c|c|c|c|c|c|}
\hline \multirow[t]{2}{*}{ Social Anxiety } & \multirow[t]{2}{*}{ Facial Emotional Changes } & \multicolumn{2}{|c|}{ Accuracy (\%) } & \multicolumn{2}{|c|}{ Response time (ms) } \\
\hline & & $M$ & $S D$ & $M$ & $S D$ \\
\hline \multirow[t]{3}{*}{ LSA } & neutral-neutral & 94.29 & 5.42 & 417.43 & 98.25 \\
\hline & angry-neutral & 84.64 & 7.66 & 419.69 & 126.09 \\
\hline & neutral-angry & 84.05 & 9.71 & 459.26 & 150.75 \\
\hline \multirow[t]{3}{*}{ HSA } & neutral-neutral & 92.05 & 5.41 & 403.33 & 129.83 \\
\hline & angry-neutral & 87.44 & 7.92 & 414.80 & 182.01 \\
\hline & neutral-angry & 83.08 & 11.60 & 440.86 & 182.98 \\
\hline
\end{tabular}




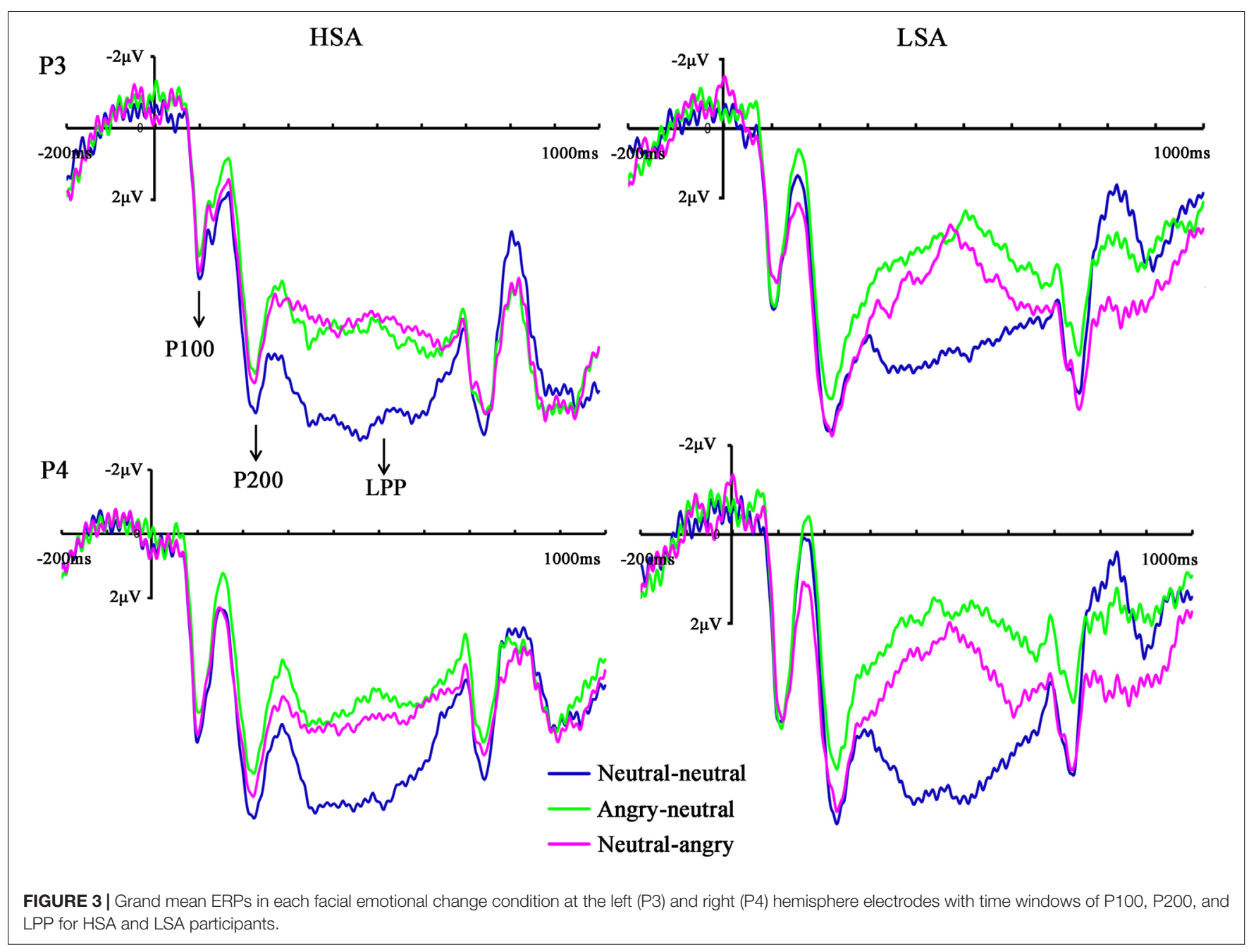

$S D=13.70 \%)$ trial $[t(26)=7.57, p<0.001)$. The RTs in the same trial were shorter $(M=398.30 \mathrm{~ms}, S D=132.16 \mathrm{~ms})$ than those seen in the different $(M=471.30 \mathrm{~ms}, S D=176.45 \mathrm{~ms})$ trial $[t(26)=-2.95, p=0.007]$. We also compared the accuracy and RT data in women vs. men facial trials to see if sex of the face stimuli had an impact. No effect reached significance [accuracy: $t(26)=1.02, p=0.319$; RTs: $t(26)=0.40$, $p=0.691]$.

\section{Electrophysiological Results P100}

The ANOVA of P100 amplitude showed that no main effects or interactions reached significance [facial emotional changes: $F(2,34)=0.49, p=0.616, \eta_{\mathrm{p}}^{2}=0.019$; hemisphere: $F(1,25)=1.93$, $p=0.177, \eta_{\mathrm{p}}^{2}=0.072$; social anxiety: $F(1,25)=0.63, p=0.434$, $\eta_{\mathrm{p}}^{2}=0.025$; hemisphere $\times$ facial emotional changes: $F(2,43)=0.33$, $p=0.688, \eta_{\mathrm{p}}^{2}=0.013$; hemisphere $\times$ social anxiety: $F(1,25)=1.99$, $p=0.171, \eta_{\mathrm{p}}^{2}=0.074 ;$ facial emotional changes $\times$ social anxiety: $F(2,34)=0.75, p=0.433, \eta_{p}^{2}=0.029$; facial emotional changes $\times$ hemisphere $\times$ social anxiety: $F(2,43)=0.09, p=0.887$, $\left.\eta_{\mathrm{p}}^{2}=0.004\right]$.
The same ANOVA on P100 latency revealed that none of the main effects or interactions were significant [facial emotional changes: $F(2,35)=0.06, p=0.881, \eta_{\mathrm{p}}^{2}=0.002$; hemisphere: $F(1,25)<0.001, p=0.991, \eta_{\mathrm{p}}^{2}<0.001$; social anxiety: $F(1,25)=0.48, p=0.496, \eta_{\mathrm{p}}^{2}=0.019$; hemisphere $\times$ facial emotional changes: $F(2,45)=1.11, p=0.332, \eta_{\mathrm{p}}^{2}=0.043$; hemisphere $\times$ social anxiety: $F(1,25)=0.39, p=0.537$, $\eta_{\mathrm{p}}^{2}=0.015$; facial emotional changes $\times$ social anxiety: $F(2,35)=2.82, p=0.090, \eta_{\mathrm{p}}^{2}=0.101 ;$ facial emotional changes $\times$ hemisphere $\times$ social anxiety: $F(2,45)=1.79, p=0.182$, $\left.\eta_{\mathrm{p}}^{2}=0.067\right]$ (Figure 3).

\section{N170}

The ANOVA of N170 amplitude revealed a significant main effect of facial emotional changes $[F(2,45)=10.96, p<0.001$, $\left.\eta_{\mathrm{p}}^{2}=0.305\right]$. Post hoc $t$-tests showed that the amplitudes (less negative amplitudes) found in the neutral-angry $(M=-0.95 \mu \mathrm{V}$, $\mathrm{SD}=4.59 \mu \mathrm{V})$ facial emotional change trial were smaller than those seen in the angry-neutral $(M=-2.17 \mu \mathrm{V}$, $S D=4.17 \mu \mathrm{V})$ facial emotional change trial $[t(26)=5.64$, $p<0.001]$, while no differences between the neutral-neutral 


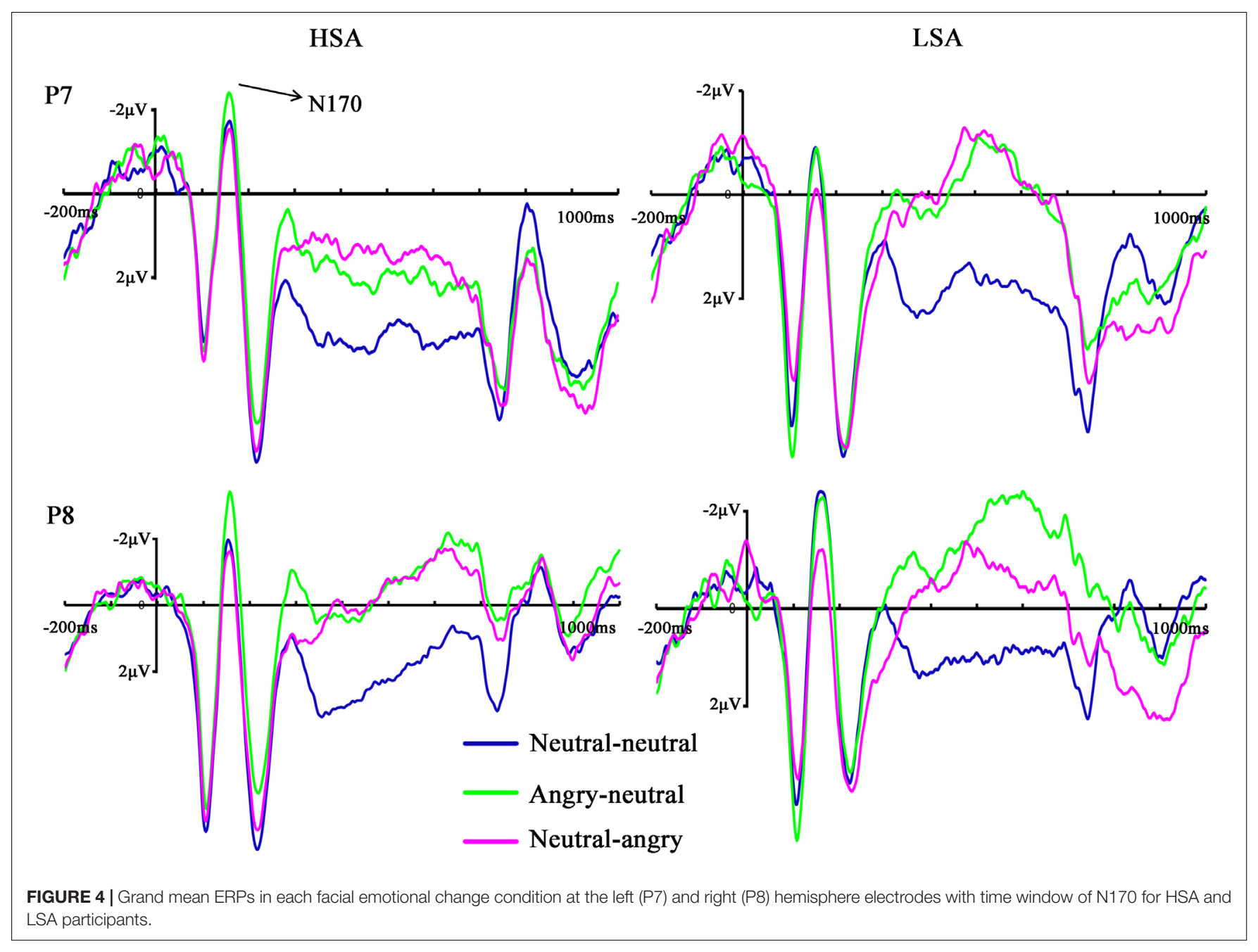

$(M=-1.70 \mu \mathrm{V}, S D=4.16 \mu \mathrm{V})$ and angry-neutral $(M=-2.17 \mu \mathrm{V}$, $S D=4.17 \mu \mathrm{V}$ ) facial emotional change trials were observed $[t(26)=1.70, p=0.101]$. Furthermore, there was a significant interaction between social anxiety and facial emotional changes $\left[F(2,45)=3.63, p=0.039, \eta_{\mathrm{p}}^{2}=0.127\right]$. Follow-up analyses confirmed the that amplitudes (more negative amplitudes) in the angry-neutral facial emotional change trial were larger than those seen in the neutral-neutral facial emotional change trial for HSA participants [angry-neutral: $M=-2.73 \mu \mathrm{V}, S D=4.76 \mu \mathrm{V}$; neutral-neutral: $M=-1.63 \mu \mathrm{V}, S D=4.57 \mu \mathrm{V} ; t(12)=-3.28$, $p=.007$ ] but not for LSA [angry-neutral: $M=-1.65 \mu \mathrm{V}$, $S D=3.66 \mu \mathrm{V}$; neutral-neutral: $M=-1.78 \mu \mathrm{V}, S D=3.91 \mu \mathrm{V}$; $t(13)=0.32, p=0.751$ ] participants (Figures 4, 5A).

The same ANOVA was performed on N170 latency. We observed a significant main effect of facial emotional changes $\left[F(2,43)=4.60, p=0.019, \eta_{\mathrm{p}}^{2}=0.156\right]$. Post hoc $t$-tests showed that the peak latency was significantly longer in the angry-neutral facial emotional change trial $(M=156.79 \mathrm{~ms}$, $S D=13.21 \mathrm{~ms}$ ) than those seen in the neutral-neutral $(M=153.64 \mathrm{~ms}, S D=12.82 \mathrm{~ms})$ case $[t(26)=-3.63, p=0.001]$. However, the differences of peak latency between the angryneutral $(M=156.79 \mathrm{~ms}, S D=13.21 \mathrm{~ms})$ and neutral-angry
$(M=154.37 \mathrm{~ms}, S D=13.10 \mathrm{~ms})$ facial emotional change trials failed to reach significance $[t(26)=-1.95, p=0.062]$. No other effect reached significance (all $F s<2.09$, $p$ s $>0.161$ ).

\section{P200}

The ANOVA of posterior P200 amplitude showed a main effect of facial emotional changes $[F(2,43)=7.20, p=0.003$, $\left.\eta_{\mathrm{p}}^{2}=0.224\right]$. Post hoc $t$-tests revealed that the P200 amplitudes were smaller in the angry-neutral facial emotional change condition $(M=9.10 \mu \mathrm{V}, S D=6.66 \mu \mathrm{V})$ than those seen in the neutral-neutral angry-neutral facial emotional change condition $(M=10.25 \mu \mathrm{V}, S D=6.54 \mu \mathrm{V})$ case $[t(26)=4.21, p<0.001]$, while no differences between the neutral-neutral $(M=10.25 \mu \mathrm{V}$, $S D=6.54 \mu \mathrm{V})$ and neutral-angry $(M=10.02 \mu \mathrm{V}, S D=7.31 \mu \mathrm{V})$ facial emotional change trials were found $[t(26)=-0.61$, $p=0.549]$. In addition, there was a significant interaction between social anxiety and hemisphere $[F(1,25)=6.20, p=0.02$, $\left.\eta_{\mathrm{p}}^{2}=0.199\right]$. The increased P200 amplitudes were observed over the left hemisphere $(M=9.13 \mu \mathrm{V}, S D=4.84 \mu \mathrm{V})$ when compared with the right $(M=7.66 \mu \mathrm{V}, S D=4.26 \mu \mathrm{V})$ hemisphere for LSA participants $[t(13)=3.00, p=0.010]$. More importantly, a significant three-way interaction among 
A $\quad \mathrm{N} 170$

LSA

HSA

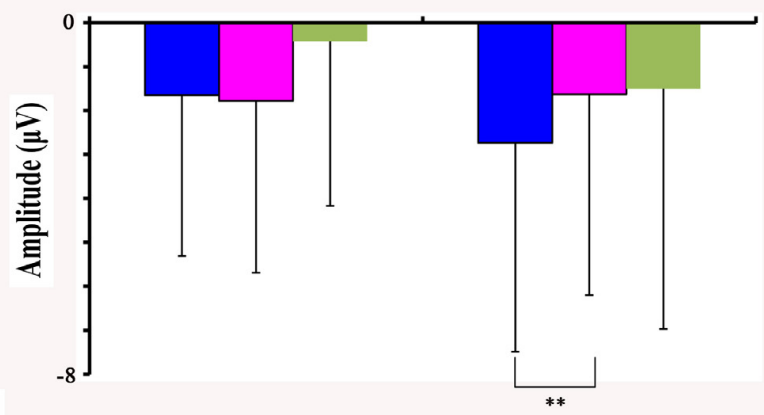

-Angry-neutral

口Neutra1-neutral

- Neutra1-angry

B P200
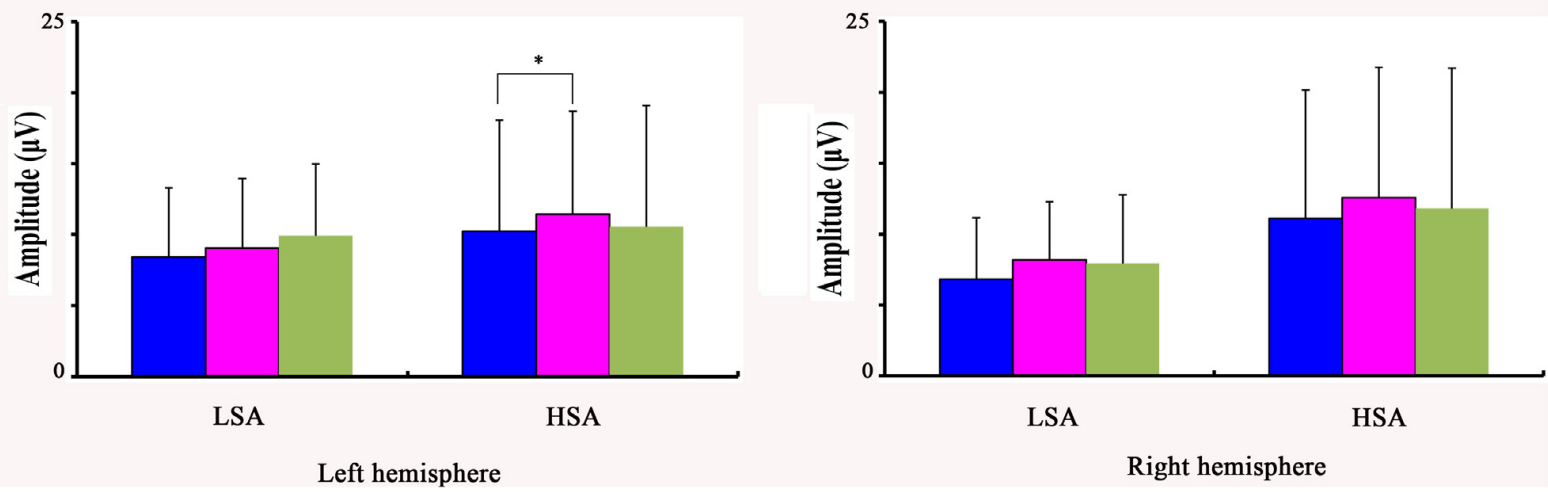

FIGURE 5 | The average N170 peaks in each facial emotional change condition for HSA and LSA participants (A). The average P200 peaks in each facial emotional change condition for HSA and LSA participants at the left and right hemisphere electrodes (B) $\left.{ }^{*} p<0.05,{ }^{* *} p<0.01\right)$.

facial emotional changes, social anxiety, and hemisphere was observed $\left[F(2,47)=3.38, p=0.045, \eta_{\mathrm{p}}^{2}=0.119\right]$. Further analyses suggested that P200 amplitudes at the left hemisphere were reduced in the angry-neutral facial emotional change trial when compared with the neutral-neutral facial emotional change trial for HSA participants [angry-neutral: $M=10.23 \mu \mathrm{V}$, $\mathrm{SD}=7.83 \mu \mathrm{V}$; neutral-neutral: $M=11.44 \mu \mathrm{V}, S D=7.24 \mu \mathrm{V}$; $t(12)=-2.35, p=0.037]$ but not for LSA participants [(angryneutral: $M=8.43 \mu \mathrm{V}, S D=4.85 \mu \mathrm{V}$; neutral-neutral: $M=9.05 \mu \mathrm{V}$, $S D=4.88 \mu \mathrm{V} ; t(13)=-1.83, p=0.090]$ (Figure 5B).

The same ANOVA when performed on posterior P200 latency showed no significant main effects or interactions [facial emotional changes: $F(2,46)=0.02, p=0.972, \eta_{\mathrm{p}}^{2}=0.001$; hemisphere: $F(1,25)=0.08, p=0.785, \eta_{\mathrm{p}}^{2}=0.003$; social anxiety: $F(1,25)=0.28, p=0.604, \eta_{\mathrm{p}}^{2}=0.011$; hemisphere $\times$ facial emotional changes: $F(2,49)=0.48, p=0.619, \eta_{\mathrm{p}}^{2}=0.019$; hemisphere $\times$ social anxiety: $F(1,25)=0.48, p=0.494$, $\eta_{\mathrm{p}}^{2}=0.019 ;$ facial emotional changes $\times$ social anxiety: $F(2,46)=0.01, p=0.996, \eta_{\mathrm{p}}^{2}<0.001 ;$ facial emotional changes $\times$ hemisphere $\times$ social anxiety: $F(2,49)=1.57, p=0.217$, $\left.\eta_{\mathrm{p}}^{2}=0.059\right]$.

\section{LPP}

The ANOVA for LPP mean amplitudes yielded a significant main effect of facial emotional changes $[F(2,41)=4.33, p=0.019$, $\left.\eta_{\mathrm{p}}^{2}=0.147\right]$. Post hoc $t$-tests revealed that the amplitudes were smaller in the neutral-angry $[M=3.21 \mu \mathrm{V}, S D=2.95 \mu \mathrm{V}$; $t(26)=2.71, p=0.012]$ and angry-neutral facial emotional change trials $[M=3.72 \mu \mathrm{V}, S D=2.69 \mu \mathrm{V} ; t(26)=2.87, p=0.008]$ than those seen in the neutral-neutral $[M=4.90 \mu \mathrm{V}, S D=2.44 \mu \mathrm{V}]$ case. No other significant amplitude differences were found for the LPP component (all Fs $<3.77$, ps $>0.064$ ). The grand average ERP waveforms and topographies of the P100, N170, P200, and LPP components in each condition are shown in Figures 3, 4, 6, 7.

\section{DISCUSSION}

The present study employed the S1-S2 paradigm that some researchers had adopted previously (Ran et al., 2014a, c; Li et al., 2017) to explore the perception of facial emotional changes in individuals with social anxiety. Our behavioral results demonstrated lower accuracy rates in the angry-neutral facial emotional change trial than those seen in the neutralneutral case. For the ERP results, we found that the N170 amplitudes were significantly larger for angry-neutral facial emotional changes than those for the neutral-neutral case only for HSA participants. However, it was found that the P200 left hemisphere amplitudes were reduced in the angry-neutral facial emotional change trial when compared with the neutral-neutral facial emotional change trial for HSA participants but not for 


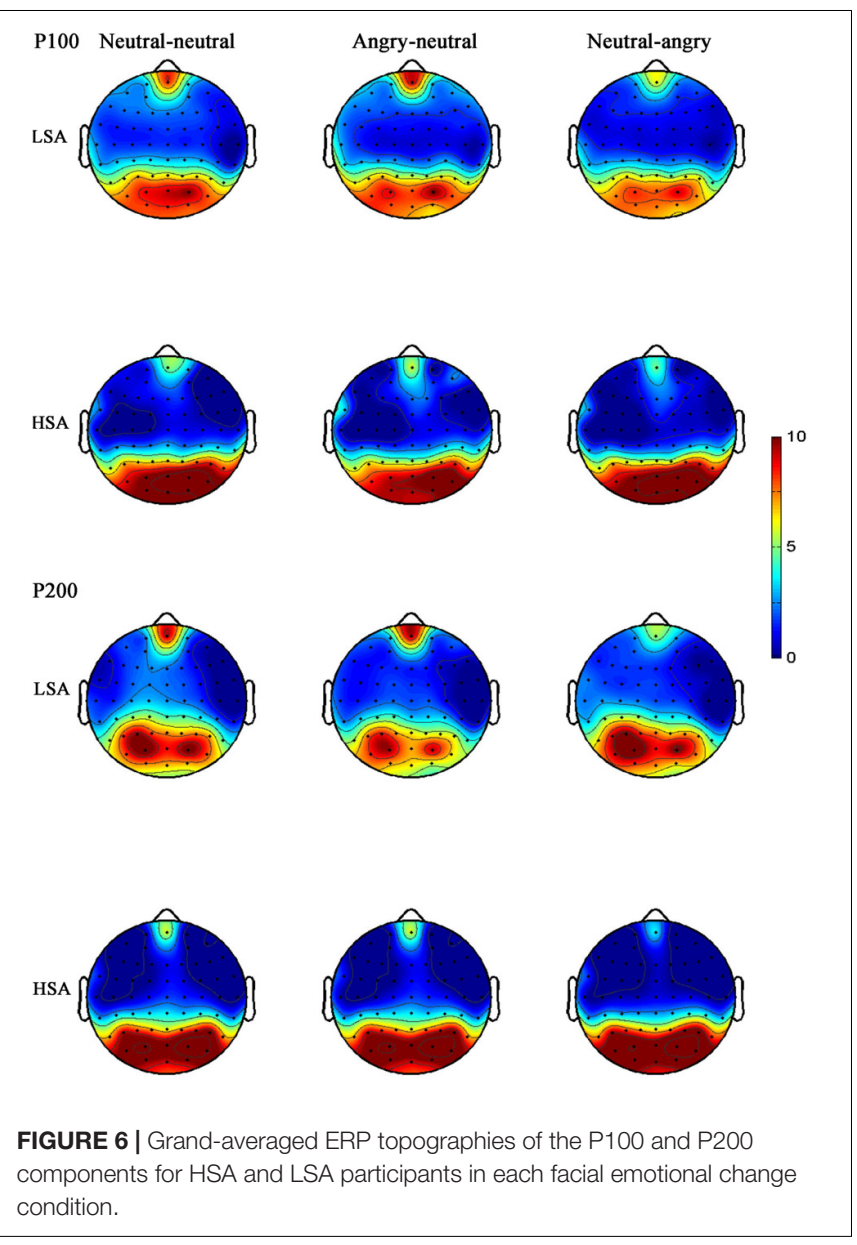

LSA participants. Finally, the LPP amplitudes in the neutralangry and angry-neutral facial emotional change trials were smaller than those in the neutral-neutral facial emotional change trials.

The present study revealed lower accuracy rates for angry-neutral facial emotional changes than those for the neutral-neutral facial emotional changes, suggesting an inferior processing of angry-neutral facial emotional changes. Considering the fact that the angry-neutral facial emotional changes are morphed (ambiguous) negative expressions (Gutiérrez-García and Calvo, 2017), people may be more cautious towards them. However, there are other studies to show that people have better performances (better memory) for emotional faces when compared with neutral faces, which is inconsistent with our finding (Grady et al., 2007). While caution should be taken in interpreting these results, one reason for the discrepancy might be that different participants were recruited in our study (HSA and LSA participants elevated on the social anxiety scale) and in the study conducted by Grady et al. (2007) (adults without a history of anxiety disorders).

The N170 component is thought to reflect the analytical processing of different facial elements (eyes, mouth, etc.) (Peschard et al., 2013). The present study found that HSA participants showed larger N170 amplitudes in angry-neutral

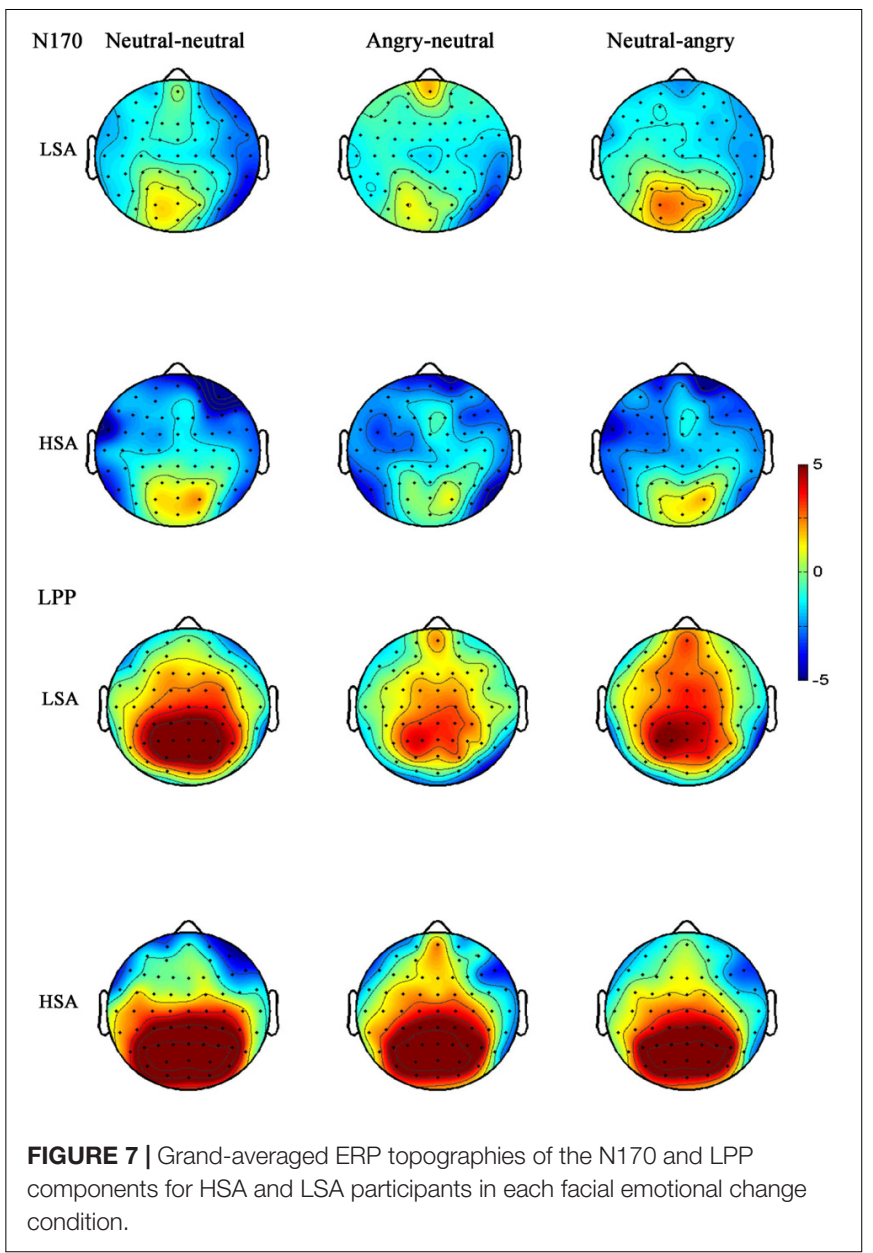

facial emotional change trials than those in the neutralneutral case. Such enhanced N170 amplitudes indicate that HSA participants may engage in more analytical processing of different facial elements when viewing the angry-neutral facial emotional changes. As HSA participants usually experience social inhibition, they have less experience with social threat stimuli (Ran and Chen, 2017). There has been a growing recognition that less experience with social stimuli leads to a relatively more feature-based processing of these stimuli (Hugenberg et al., 2007; Short and Mondloch, 2013). Similar to our finding, larger N170 amplitudes were observed when persons clinically diagnosed with social phobia (social phobic persons) processed static angry faces in an emotion identification task (Kolassa and Miltner, 2006).

Interestingly, the P200 was functionally associated with the evaluation of emotional relevance of a visual stimulus, as reported by many studies previously (Kolassa et al., 2009; Peschard et al., 2013). The P200 amplitudes were decreased in the angry-neutral facial emotional change trials when compared with neutralneutral facial emotional change trials for HSA participants but not for LSA participants, which suggests that HSA participants may have difficulties in processing emotions when they encounter angry-neutral facial emotional changes. This is well in line with evidence from studies on emotional processes that indicate the deficient emotion evaluation in high anxious subjects (e.g., 
Rossignol et al., 2005). The difficulties with emotion evaluation in HSA participants might arise from their distorted appraisals of social situations, which is corroborated by the fact that these participants tend to show inaccurate interpretations of the self (socially incompetent) and others (critical judges) (Goldin et al., 2009). However, increased P2 amplitudes were reported in HSA participants for static angry faces (Ran and Chen, 2017), which was inconsistent with our findings. Such inconsistency suggests that facial expression (dynamic and static expressions) may moderate N170 in HSA participants. This needs to be examined in further studies.

The present result found smaller LPP amplitudes in the neutral-angry facial emotional change trial than those in the neutral-neutral case. This is similar to the previous ERP studies (Yuan et al., 2007; Chen and Luo, 2010), which demonstrated that reduced LPP (or P3) amplitudes were elicited by static negative stimuli. However, some other studies found that negative pictures evoked enhanced LPP amplitudes (Moser et al., 2008; Wieser and Moscovitch, 2015). Yuan et al. (2007) suggested that the reduced LPP (or P3) amplitudes under negative condition were due to the fact that participants needed to overcome the emotional negativity bias that favors the processing of negatively valenced information in implicit emotional experiments. In addition, the LPP amplitudes in the angry-neutral facial emotional change trials were smaller than those in the neutral-neutral facial emotional change trials. This suggests that the differences between the neutral-angry and angry-neutral facial emotional changes were not observed at a later stage of processing. However, the N170 (an early component) amplitudes were found to be smaller in the neutral-angry facial emotional change trials than those in the angry-neutral case.

Some studies have employed the S1-S2 paradigm to explore the recognition of emotional faces (Ran et al., 2014a,c; Li et al., 2017). Given that the processing of emotion was implicit (judging the identity of the faces or identifying the gender of the faces) in these studies, such task manipulation is not optimal. It would be nice to adopt an explicit task of emotion (judging the expression/expression change) in further research.

\section{REFERENCES}

Beck, A. T., and Beamesderfer, A. (1974). Assessment of depression: the depression inventory. Mod. Probl. Pharmacopsychiatry 7, 151-169. doi: 10.1159/000395074

Bentin, S., Allison, T., Puce, A., Perez, E., and McCarthy, G. (1996). Electrophysiological studies of face perception in humans. J. Cogn. Neurosci. 8, 551-565. doi: 10.1162/jocn.1996.8.6.551

Caharel, S., Montalan, B., Fromager, E., Bernard, C., Lalonde, R., and Mohamed, R. (2011). Other-race and inversion effects during the structural encoding stage of face processing in a race categorization task: an event-related brain potential study. Int. J. Psychophysiol. 79, 266-271. doi: 10.1016/j.ijpsycho.2010. 10.018

Campanella, S., Montedoro, C., Streel, E., Verbanck, P., and Rosier, V. (2006). Early visual components (P100, N170) are disrupted in chronic schizophrenic patients: an event-related potentials study. Clin. Neurophysiol. 36, 71-78. doi: 10.1016/j.neucli.2006.04.005

Chen, C. P., and Luo, Y. J. (2010). Attentional negativity bias moderated by positive mood arousal. Sci. Bull. 55, 2010-2015. doi: 10.1007/s11434-0103220-6

\section{CONCLUSION}

In the present study, the ERP measure was used to explore the perception of facial emotional changes in individuals with social anxiety. The behavioral data showed lower accuracy rates in the angry-neutral facial emotional change trial than those in the neutral-neutral case, indicating an inferior processing of the angry-neutral facial emotional change. The electrophysiological data showed larger N170 amplitudes in the angry-neutral facial emotional change trial than those in the neutral-neutral case for HSA participants, implying that they might be engaged in more analytical processing of different facial elements. However, HSA participants showed smaller P200 amplitudes in the angry-neutral facial emotional change trial when compared with the neutral-neutral case, which suggested that they might have difficulties in processing emotions when they encounter angry-neutral facial emotional changes. Finally, we found that the LPP amplitudes in the neutral-angry and angry-neutral facial emotional change trials were smaller than those in the neutral-neutral facial emotional change trials, regardless of the social anxiety. In short, we suggest that the effect of social anxiety on the facial emotional changes may change over time.

\section{AUTHOR CONTRIBUTIONS}

QZ designed the experiments. QZ and GR collected and analyzed the data. QZ primarily wrote the manuscript. All authors discussed the results and commented on the manuscript.

\section{FUNDING}

This research was supported by the Person of Outstanding Ability of China West Normal University (Grant No. 17YC210) and the Social Science Research "the 13th FiveYear Plan" Project for Nanchong in 2017 year (Grant No. NC2017C057).

Chen, X., Ran, G., Zhang, Q., and Hu, T. (2015). Unconscious attention modulates the silencing effect of top-down predictions. Conscious. Cogn. 34, 63-72. doi: 10.1016/j.concog.2015.03.010

Fishman, I., Ng, R., and Bellugi, U. (2012). Neural processing of race by individuals with williams syndrome: do they show the other-race effect? (And why it matters). Soc. Neurosci. 7:373. doi: 10.1080/17470919.2011.628759

Grady, C. L., Hongwanishkul, D., Keightley, M., Lee, W., and Hasher, L. (2007). The effect of age on memory for emotional faces. Neuropsychology 21, 371-380. doi: 10.1037/0894-4105.21.3.371

Goldin, P. R., Manber, T., Hakimi, S., Canli, T., and Gross, J. J. (2009). Neural bases of social anxiety disorder: emotional reactivity and cognitive regulation during social and physical threat. Arch. Gen. Psychiatry 66, 170-180. doi: 10. 1001/archgenpsychiatry.2008.525

Gutiérrez-García, A., and Calvo, M. G. (2017). Social anxiety and threat-related interpretation of dynamic facial expressions: sensitivity and response bias. Pers. Individ. Differ. 107, 10-16. doi: 10.1016/j.paid.2016.11.025

Hagemann, J., Straube, T., and Schulz, C. (2016). Too bad: bias for angry faces in social anxiety interferes with identity processing. Neuropsychologia 84, 136-149. doi: 10.1016/j.neuropsychologia.2016.02.005 
Heuer, K., Lange, W.-G., Isaac, L., Rinck, M., and Becker, E. S. (2010). Morphed emotional faces: emotion detection and misinterpretation in social anxiety. J. Behav. Ther. Exp. Psychiatry 41, 418-425. doi: 10.1016/j.jbtep.2010.04.005

He, Y., and Zhang, M. (2004). Psychometric investigation of the liebowitz social anxiety scale. J. Diagn. Concepts Pract. 3, 89-93.

Hinojosa, J. A., Mercado, F., and Carretié, L. (2015). N170 sensitivity to facial expression: a meta-analysis. Neurosci. Biobehav. Rev. 55, 498-509. doi: 10.1016/ j.neubiorev.2015.06.002

Hugenberg, K., Miller, J., and Claypool, H. M. (2007). Categorization and individuation in the cross-race recognition deficit: toward a solution to an insidious problem. J. Exp. Soc. Psychol. 43, 334-340. doi: 10.1016/j.jesp.2006. 02.010

Iverach, L., and Rapee, R. M. (2014). Social anxiety disorder and stuttering: current status and future directions. J. Fluency Disord. 40, 69-82. doi: 10.1016/j.jfludis. 2013.08.003

Kirsch, P. (2015). Oxytocin in the socioemotional brain: implications for psychiatric disorders. Dialogues Clin. Neurosci. 17, 463-476.

Kolassa, I. T., and Miltner, W. H. (2006). Psychophysiological correlates of face processing in social phobia. Brain Res. 1118, 130-141. doi: 10.1016/j.brainres. 2006.08.019

Kolassa, I. T., Kolassa, S., Bergmann, S., Lauche, R., Dilger, S., Miltner, W. H. R., et al. (2009). Interpretive bias in social phobia: an ERP study with morphed emotional schematic faces. Cogn. Emot. 23, 69-95. doi: 10.1080/ 02699930801940461

Kross, E., Bruehlmansenecal, E., Park, J., Burson, A., Dougherty, A., Shablack, H., et al. (2014). Self-talk as a regulatory mechanism: how you do it matters. J. Pers. Soc. Psychol. 106, 304-324. doi: 10.1037/a0035173

Joormann, J., and Gotlib, I. H. (2006). Is this happiness i see? Biases in the identification of emotional facial expressions in depression and social phobia. J. Abnorm. Psychol. 115, 705-714. doi: 10.1037/0021-843X.115.4.705

Li, D., Yu, F., Ye, R., Chen, X., Xie, X., Zhu, C., et al. (2017). How does gaze direction affect facial processing in social anxiety? An ERP study. Psychiatry Res. 251, 155-161. doi: 10.1016/j.psychres.2017.02.018

Luo, W., Feng, W., He, W., Wang, N. Y., and Luo, Y. J. (2010). Three stages of facial expression processing: ERP study with rapid serial visual presentation. Neuroimage 49, 1857-1867. doi: 10.1016/j.neuroimage.2009.09.018

Mathew, A. R., Pettit, J. W., Lewinsohn, P. M., Seeley, J. R., and Roberts, R. E. (2011). Co-morbidity between major depressive disorder and anxiety disorders: shared etiology or direct causation? Psychol. Med. 41, 2023-2034. doi: 10.1017/ S0033291711000407

Moser, J. S., Huppert, J. D., Duval, E., and Simons, R. F. (2008). Face processing biases in social anxiety: an electrophysiological study. Biol. Psychol. 78, 93-103. doi: 10.1016/j.biopsycho.2008.01.005

Mühlberger, A., Wieser, M. J., Herrmann, M. J., Weyers, P., Tröger, C., and Pauli, P. (2009). Early cortical processing of natural and artificial emotional faces differs between lower and higher socially anxious persons. J. Neural Transm. 116, 735-746. doi: 10.1007/s00702-008-0108-6

Peschard, V., Philippot, P., Joassin, F., and Rossignol, M. (2013). The impact of the stimulus features and task instructions on facial processing in social anxiety: an ERP investigation. Biol. Psychol. 93, 88-96. doi: 10.1016/j.biopsycho.2013. 01.009

Pierce, T. (2009). Social anxiety and technology: face-to-face communication versus technological communication among teens. Comput. Hum. Behav. 25, 1367-1372. doi: 10.1016/j.chb.2009.06.003

Ran, G., and Chen, X. (2017). The impact of top-down prediction on emotional face processing in social anxiety. Front. Psychol. 8:1269. doi: 10.3389/fpsyg.2017. 01269

Ran, G., Chen, X., and Pan, Y. (2014a). Human sex differences in emotional processing of own-race and other-race faces. Neuroreport 25, 683-687. doi: 10.1097/WNR.0000000000000158

Ran, G. M., Chen, X., Pan, Y. G., Hu, T. Q., and Ma, J. (2014b). Effects of anticipation on perception of facial expressions. Percept. Mot. Skills 118, 195-209. doi: 10.2466/24.PMS.118k13w4
Ran, G., Zhang, Q., Chen, X., and Pan, Y. (2014c). The effects of prediction on the perception for own-race and other-race faces. PLoS One 9:e114011. doi: 10.1371/journal.pone.0114011

Rossignol, M., Philippot, P., Bissot, C., Rigoulot, S., and Campanella, S. (2012). Electrophysiological correlates of enhanced perceptual processes and attentional capture by emotional faces in social anxiety. Brain Res. 1460, 50-62. doi: 10.1016/j.brainres.2012.04.034

Rossignol, M., Philippot, P., Douilliez, C., Crommelinck, M., and Campanella, S. (2005). The perception of fearful and happy facial expression is modulated by anxiety: an event-related potential study. Neurosci. Lett. 377, 115-120. doi: 10.1016/j.neulet.2004.11.091

Schendan, H. E., Ganis, G., and Kutas, M. (1998). Neurophysiological evidence for visual perceptual categorization of words and faces within $150 \mathrm{~ms}$. Psychophysiology 35, 240-251. doi: 10.1111/1469-8986.3530240

Sheehan, K. A., Mcarthur, G. M., and Bishop, D. V. M. (2005). Is discrimination training necessary to cause changes in the P2 auditory event-related brain potential to speech sounds? Cognit. Brain Res. 25, 547-553. doi: 10.1016/j. cogbrainres.2005.08.007

Short, L. A., and Mondloch, C. J. (2013). Aging faces and aging perceivers: young and older adults are less sensitive to deviations from normality in older than in young adult faces. Perception 42, 795-812. doi: 10.1068/p7380

Spielberger, C. D., Gorsuch, R. L., Lusthene, R., Vagg, P. R., and Jacobs, G. A. (1983). Manual for the State-Trait Anviety Inventory. Palo Alto, CA: Consulting Psychologists Press.

Sje, L., and van Strien, J. W. (2018). Early visual processing of snakes and angry faces: an ERP study. Brain Res. 1678, 297-303. doi: 10.1016/j.brainres.2017.10. 031

Taylor, M., and Khan, S. (2000). Top-down modulation of early selective attention processes in children. Int. J. Psychophysiol. 37, 135-147. doi: 10.1016/S01678760(00)00084-2

Van Beek, L., Ghesquièr, P., De Smedt, B., and Lagae, L. (2014). The arithmetic problem size effect in children: an event-related potential study. Front. Hum. Neurosci. 8:756. doi: 10.3389/fnhum.2014.00756

Wieser, M. J., and Moscovitch, D. A. (2015). The effect of affective context on visuocortical processing of neutral faces in social anxiety. Front. Psychol. 6:1824. doi: 10.3389/fpsyg.2015.01824

Wieser, M. J., Pauli, P., Reicherts, P., and Mühlberger, A. (2010). Don't look at me in anger! Enhanced processing of angry faces in anticipation of public speaking. Psychophysiology 47, 271-280. doi: 10.1111/j.1469-8986.2009.00938.x

Yuan, J., Zhang, Q., Chen, A., Li, H., Wang, Q., Zhuang, Z., et al. (2007). Are we sensitive to valence differences in emotionally negative stimuli? Electrophysiological evidence from an ERP study. Neuropsychologia 45, 27642771. doi: 10.1016/j.neuropsychologia.2007.04.018

Zhang, P., Chen, X., Yuan, P., Zhang, D., and He, S. (2006). The effect of visuospatial attentional load on the processing of irrelevant acoustic distractors. Neuroimage 33, 715-724. doi: 10.1016/j.neuroimage.2006.07.015

Zhang, Q., Ran, G., and Yin, T. (2017). The perception of own-race and other-race faces in emotional changes. Chin. J. Clin. Psychol. 25, 608-612. doi: 10.16128/j. cnki.1005-3611.2017.04.004

Zhang, Q., Yin, T., and Ran, G. (2015). Psychological and neural mechanisms for the superiority effect of dynamic facial expressions. Adv. Psychol. Sci. 23, 1514-1522. doi: 10.3724/SP.J.1042.2015.01514

Conflict of Interest Statement: The authors declare that the research was conducted in the absence of any commercial or financial relationships that could be construed as a potential conflict of interest.

Copyright (c) 2018 Zhang, Ran and Li. This is an open-access article distributed under the terms of the Creative Commons Attribution License (CC BY). The use, distribution or reproduction in other forums is permitted, provided the original author(s) and the copyright owner(s) are credited and that the original publication in this journal is cited, in accordance with accepted academic practice. No use, distribution or reproduction is permitted which does not comply with these terms. 